Article

\title{
The Visual Vaccine Debate on Twitter: A Social Network Analysis
}

\author{
Elena Milani ${ }^{1, *}$, Emma Weitkamp ${ }^{1}$ and Peter Webb ${ }^{2}$ \\ ${ }^{1}$ Department of Applied Sciences, University of the West of England, Bristol, BS16 1QY, UK; \\ E-Mails: elena.milani@uwe.ac.uk (E.M.), emma.weitkamp@uwe.ac.uk (E.W.) \\ 2 Department of Health and Social Sciences, University of the West of England, Bristol, BS16 1QY, UK; \\ E-Mail: peter.webb@uwe.ac.uk \\ * Corresponding author
}

Submitted: 29 January 2020 | Accepted: 18 April 2020 | Published: 26 June 2020

\begin{abstract}
Pro- and anti-vaccination users use social media outlets, such as Twitter, to join conversations about vaccines, disseminate information or misinformation about immunization, and advocate in favour or against vaccinations. These users not only share textual content, but also images to emphasise their messages and influence their audiences. Though previous studies investigated the content of vaccine images, there is little research on how these visuals are distributed in digital environments. Therefore, this study explored how images related to vaccination are shared on Twitter to gain insight into the communities and networks formed around their dissemination. Moreover, this research also investigated who influences the distribution of vaccine images, and could be potential gatekeepers of vaccination information. We conducted a social network analysis on samples of tweets with images collected in June, September and October 2016. In each dataset, proand anti-vaccination users formed two polarised networks that hardly interacted with each other, and disseminated images among their members differently. The anti-vaccination users frequently retweeted each other, strengthening their relationships, making the information redundant within their community, and confirming their beliefs against immunisation. The pro-vaccine users, instead, formed a fragmented network, with loose but strategic connections that facilitated networking and the distribution of new vaccine information. Moreover, while the pro-vaccine gatekeepers were non-governmental organisations or health professionals, the anti-vaccine ones were activists and/or parents. Activists and parents could potentially be considered as alternative but trustworthy sources of information enabling them to disseminate misinformation about vaccinations.
\end{abstract}

\section{Keywords}

activism; misinformation; social media; social network analysis; Twitter; vaccination

\section{Issue}

This article is part of the issue "Health and Science Controversies in the Digital World: News, Mis/Disinformation and Public Engagement" edited by An Nguyen (Bournemouth University, UK) and Daniel Catalan (University Carlos III of Madrid, Spain).

(C) 2020 by the authors; licensee Cogitatio (Lisbon, Portugal). This article is licensed under a Creative Commons Attribution 4.0 International License (CC BY).

\section{Introduction}

This study explored the dynamics of the dissemination of vaccination images on Twitter to gain insights into the pro- and anti-vaccine networks sharing them. Online images can increase the sharing rate and visibility of tweets (Chen \& Dredze, 2018) and can be used to articulate the messages in the text of the tweet or to elicit emotive response (Giglietto \& Lee, 2017). Moreover, images can convey health messages effectively (Houts, Doak, Doak, \& Loscalzo, 2006) and influence public opinion toward health issues (Apollonio \& Malone, 2009). Previous studies on vaccine images analysed their sentiment and content (Chen \& Dredze, 2018; Guidry, Carlyle, Messner, \& Jin, 2015; Lama et al., 2018); however, none of them investigated how this visual information is disseminated 
online. Therefore, this study explored whether vaccination images flow within or between insular communities, how they are shared within the same network, and who the most influential anti- and pro-vaccine actors are. These actors could influence the distribution of vaccine images and could be potential gatekeepers of vaccination information.

This research focuses on Twitter since it is a news feed where most users' profiles and their messages are public and thus accessible for research purposes (Kumar, Morstatter, \& Liu, 2013; Kwak, Lee, Park, \& Moon, 2010). Moreover, Twitter allows thematic conversations to emerge between either friends or strangers by sharing tweets, which are short textual messages of 280 characters that can include multimedia content such as pictures, gifs, videos, URLs, and geotags. The voluntary sharing of information and personal opinions online by Internet users provides the opportunity to understand more deeply audiences' understanding, attitudes, and beliefs towards specific topics, such as health (Scanfeld, Scanfeld, \& Larson, 2010; Wilson, Atkinson, \& Deeks, 2014) and scientific controversies, including vaccines (Love, Himelboim, Holton, \& Stewart, 2013).

The information posted on Twitter is not necessarily filtered by traditional gatekeepers (e.g., journalists, press officers), thus giving the opportunity to scientists, activists, and other individuals to reach their audience directly. For example, a health practitioner could engage with their public informally and directly on Twitter instead of using official channels (e.g., health organisation's website; Schmidt, 2014). By bypassing the information gatekeeping system, Twitter and other social media outlets allowed access to a variety of traditional and alternative sources of information (Murthy, 2012). However, this also facilitated the dissemination of misinformation online. False news items are found to spread faster and more widely on Twitter than true news, especially those about politics (Vosoughi, Roy, \& Aral, 2018). However, most political exposure is still from reliable sources of information, and only a small fraction of users shares news from disinformation sources (Grinberg, Joseph, Friedland, Swire-Thompson, \& Lazer, 2019). In the case of vaccinations, anti-vaccine users were also found to be a minority on Twitter (Bello-Orgaz, Hernandez-Castro, \& Camacho, 2017) that share alternative sources of information rather than traditional ones (Himelboim, Xiao, Lee, Wang, \& Borah, 2019). These actors may use social media to disseminate information (and misinformation) about vaccines and sensationalise objections to vaccinations (Witteman \& Zikmund-Fisher, 2012). This enables them to reach their target audience directly and potentially influence their risk perception toward vaccines, thus persuading them not to vaccinate themselves and/or their children (Betsch, Renkewitz, Betsch, \& Ulshöfer, 2010).

\section{Literature Review}

\section{1. 'Alternative' Experts and Disseminating (Mis)Information}

The Internet and social media outlets allow easy access to information; any Internet user can consume and produce textual and visual content and potentially reach their target audience online. Hence, these actors can communicate with their public directly on digital media, bypassing the traditional gatekeeping system of news media and journalists (Schmidt, 2014). They can reach blog readers or social media followers, who already know about them and are interested in their content. They can also reach organic or ad hoc publics, who come across their content by searching Google or following Twitter hashtags (Bruns \& Moe, 2014). Either way, Internet users that regularly contribute to a conversation on a topic with high quality information tend to be acknowledged as 'experts' by the other participants (Bruns, 2008). For example, a parent sharing good content about vaccinations frequently with a vaccine group online could be considered an alternative expert about vaccinations by the other members of the group. On Twitter, conversations formed around hashtags can become communities. In this case, every user tweeting with a certain hashtag regularly can be considered a member of that community (Bruns \& Burgess, 2015). Some of these users may become experts, and even opinion leaders if they have several followers and strategic connections within the community that allow them to control the flow of information. Opinion leaders would be able to decide what content to share (or not) with the members, thus potentially influencing the community's common opinion (Murthy, 2012).

The type of content valued may differ among communities. For example, in polarised communities, members do not share information that does not support the beliefs of the community. This can reinforce their confirmation bias and misconceptions or misunderstandings of scientific content (All Europe Academies, 2019), as in the case of anti-vaccine communities that only share information supporting their claims while excluding any scientific evidence claiming the opposite (Kata, 2012). By filtering content that favours a certain perspective, polarised communities can facilitate the spreading of misinformation and conspiracy theories among their members (Del Vicario et al., 2016). In these groups, experts and gatekeepers may not be scientists and journalists, and the quality of their contributions may not be valued based on scientific accuracy, but on agreement with the members' opinions. Moreover, members of polarised communities tend to have a negative perception of outsiders, and do not acknowledge the authority of external experts, even those whose expertise is recognised by the scientific community (Bruns, 2008; Southwell, 2013). 


\subsection{Anti-Vaccine Activism and Misinformation on Twitter}

Though vaccines have eradicated or significantly reduced vaccine-preventable diseases, and are considered one of the most effective public health interventions, they have aroused public concerns about their safety and effectiveness since first proposed. Moreover, the vaccine controversy has been recently stimulated by a range of factors (e.g., occurrence of vaccine side effects, scepticism or non-acceptance of scientific evidence; Larson, Cooper, Eskola, Katz, \& Ratzan, 2011), which have been highlighted by anti-vaccine movements (Dubé, Vivion, \& MacDonald, 2015).

Anti-vaccine movements disseminate their alternative information on the Internet and social media. Previous research on Twitter found that most of the antivaccination messages claim that vaccines are dangerous and encourage vaccine refusal. These tweets share personal stories, anecdotes, opinions, misinformation, and conspiracy theories (Dunn, Leask, Zhou, Mandl, \& Coiera, 2015; Mitra, Counts, \& Pennebaker, 2016); moreover, they tend to share links to emerging/alternative news websites rather than traditional ones (Meadows, Tang, $\&$ Liu, 2019). Though anti-vaccination tweets are only a minority in the vaccine debate on Twitter (Love et al., 2013) and their volume has decreased since 2015 while that of pro-vaccine messages has increased, the number of anti-vaccine users has doubled (Gunaratne, Coomes, \& Haghbayan, 2019). Anti-vaccine activists tend to be alternative sources of information (Himelboim et al., 2019) and believe conspiracy theories related to vaccination (Mitra et al., 2016). These actors form a polarised and tight community that does not interact with outsiders and does not engage with pro-vaccine users (Bello-Orgaz et al., 2017; Yuan \& Crooks, 2018). Antivaccine actors do not share only text and hyperlinks, but images too. Though image sharing is a popular activity online (Duggan, 2013), there is little research on vaccine images disseminated on social media (Chen \& Dredze, 2018; Guidry et al., 2015) and none of these previous studies considered how these images are distributed on Twitter, by who and to whom.

\subsection{Social Media Network Analysis}

Social media network analysis can be an effective method to study the dissemination of vaccine images on Twitter, as it investigates the distribution of tweets and retweets among and within networks and the actors that could affect this distribution (Himelboim, 2017). A Twitter network can be formed by users conversing about the same topic, using the same hashtags, and the tweets and retweets they share with each other (i.e., their connections; Kumar et al., 2013). Retweets can be reciprocal or not, thus their reciprocity and direction can provide insights on the connectivity and attitudes of a network. For example, a network could be formed by two polarised groups, highly connected within but barely connected between them. These two groups could be formed by like-minded people around opposite perspectives on the same topic, e.g., in favour or against vaccination (Smith, Rainie, Shneiderman, \& Himelboim, 2014). Another example could be a network formed by one central actor highly retweeted by the other members; in this case the central member broadcasts their message to the others, like a hub, but does not engage with them (Himelboim, Smith, Rainie, Shneiderman, \& Espina, 2017).

The connectivity of a network can also provide other insights: In a network where members frequently retweet each other but not outsiders, information will be disseminated more efficiently but it will also become redundant (Kadushin, 2011). Moreover, the dense connectivity could give a sense of trust, safety, and support to its members, but also reinforce their common beliefs and increase their negative perception of outsiders (Southwell, 2013). In a loose network, instead, the sense of support may not be strong but there would be better access to and diffusion of new information (Kadushin, 2011). Social network analysis can also be an effective means of identifying actors exerting influence on the information flow within a network (Himelboim, 2017). The central actor mentioned before, the hub, could be one of these; they regulate the types of messages and images circulating within the group. A key actor could also be a broker connecting groups that otherwise would not be linked. For example, these actors could retweet or be retweeted by different groups, thus influencing their access to new information (Kadushin, 2011; Kumar et al., 2013). In this study, we distinguish actors as individuals that can potentially control the information flow in a network, and users as generic Twitter users or members of a network.

\section{Aims and Objectives}

The aim of this study is to fill a gap in our knowledge of how vaccine images are shared on Twitter. As such, the study takes an exploratory approach to gain insights into the diversity of networks, communities, and actors involved. Specifically, the study seeks to explore how vaccine visual information (and misinformation) circulates within and among Twitter networks and to identify actors that could potentially influence the flow of vaccination images. These actors may be the same as those participating in health conversations-advocates and health professionals ( $\mathrm{Xu}, \mathrm{Chiu}, \mathrm{Chen}$, \& Mukherjee, 2015) - or may include a wide range of other actors, such as Governmental Health Agencies, NGOs, charities, the media, academics, and parents. Since the types of actor participating in the sharing of visual material may differ between anti- and pro-vaccine communities, identifying these differences will provide insights into the types of 'gatekeepers' and 'experts' that are acknowledged by each community. This has practical relevance for those seeking to participate in the visual vaccine discourse on 
Twitter. Therefore, this study focused on:

- The pro- and anti-vaccine actors that are most influential in their respective communities, identifying the types of groups they represent;

- How these actors share information within their networks.

Sharing practices may differ between actor types and the networks within which they operate. Moreover, sharing patterns can vary depending on the network structure (Himelboim et al., 2017). Therefore, analysing the dynamics of information flow within and between antiand pro-vaccine networks may provide information on how (mis)information circulates. Hence, this research also analyses:

- Whether anti- and pro-vaccine communities share visual information between each other;

- How anti- and pro-vaccine communities share images with their members.

\section{Methods}

\subsection{Data Collection, Preparation, and Classification}

This study undertakes a qualitative comparison between the dissemination of anti- and pro-vaccine images within and between Twitter networks. To explore vaccine Twitter networks and identify recurrent influential actors, we collected tweets posted in June (from 26th to 30th), September (from 9th to 13th), and October (from 4th to 11th) 2016 using the software NodeXL Pro, developed by the Social Media Research Foundation. We gathered only tweets written in English, having an image uploaded on Twitter originally and at least one of the following hashtags: \#vaccine(s), \#vaccination(s), \#immunization, \#vaccineswork, \#whylvax, \#antivax, \#CDCwhistleblower, \#vaccineinjury, \#vaxxed, and \#hearus. Hashtags are words preceded by the \# sign that label specific conversations on Twitter. To select the hashtags for the collection criteria, we first consulted the online services Symplur.com and Hashtagify.me to identify those relevant to vaccines, and then we checked on Twitter how often these hashtags were used during June 2016. We finally selected the twelve most tweeted hashtags: four generic, four anti-vaccine, and four provaccine. We did not include words (e.g., vaccine[s]) in the collection criteria since hashtags, not words, are usually used to find all published tweets with those keywords and to join the respective conversations (Bruns \& Stieglitz, 2014). Moreover, users tend to include hashtags in their posts to reach audiences interested in the topic that are not yet their followers (Bruns \& Moe, 2014). For these reasons, in this study we preferred to focus on ongoing visual conversations around hashtags.

We gathered 4480 tweets in June, 2658 tweets in September, and 5262 tweets in October. Since we were interested in how images are shared (retweeted) among vaccine networks, we considered only retweets and mentions in the social network analysis (Kumar et al., 2013). We removed tweets that were not relevant to vaccinations and tweets that were replies, obtaining final samples of 3573,1932 , and 3778 tweets, respectively. Then, to distinguish different conversations about immunisation, and therefore the networks participating in these conversations, we classified the tweets as follows:

- Anti-vaccine: Tweets strongly against vaccinations, claiming conspiracy theories, disseminating misinformation about vaccines, or opposing pro-vaccine messages; e.g., 'the CDC will never admit that \#vaccines cause autism';

- Pro-vaccine: Tweets strongly in favour of vaccinations, promoting immunisation campaigns, providing medical advice regarding vaccinations, or mocking anti-vaccine claims; e.g., '\#VaccinesWork-one step forward to end polio';

- Pro-safe vaccine: Tweets expressing concerns about vaccinations, i.e., the need for more controls and ethical considerations in vaccine production, administration, and business; e.g., 'Vaccinations should be administered only after being tested';

- News: News tweets that included text, web links, hashtags, or images that referred to newspaper, webzine or magazine news articles (opinion articles were excluded) about vaccinations, outbreaks, immunisation campaigns, vaccine research and development; e.g., 'the clinical trial for Zika vaccines has started';

- Academic: Tweets about journal papers, academic job applications, patient recruitment, or medical/academic conferences, lectures, seminars.

We followed the guidelines suggested by Braun and Clarke (2013) to code the tweets. We identified potential categories during the preliminary analysis of the tweets from the first collection (June 2016), considering tweets' content, images, hashtags, embedded links, and Twitter users. Photos, URLs, and other tweets' attachments were also considered, following LeFebvre and Armstrong's (2018) directions for content and sentiment analysis. Once we defined the classification system it was applied to all three datasets, including the first one.

\subsection{Social Network Analysis}

To analyse the sharing patterns of vaccine images, for each data collection we plotted the networks of retweets and mentions in clusters by the Clauset-Newman-Moore algorithm (Clauset, Newman, \& Moore, 2004). First, we focused on the overall networks, then we explored the anti- and pro-vaccine groups separately. For each network and group, we analysed the size of network and its connectivity (Kumar et al., 2013). For example, we considered parameters such as density (the ratio between 
the number of observed retweets and the number of possible retweets in the network) and modularity (which ranges from $0=$ the users in a network are highly connected, to 1 = they are not connected; Newman, 2010). Density and modularity can provide insights into the connectivity within the same group and among different communities and clusters. These two values must be considered together since high density can indicate a strongly connected network, which might be unified or divided depending on its low or high modularity, respectively (Himelboim, 2017). We used these four parameters to explore how the visual information flows within and among groups and clusters and its reach.

\subsection{Analysis of Key Actors}

In this research, we defined 'gatekeepers' as those Twitter actors that could potentially control the information flowing into and within a network. Moreover, we defined 'hubs' as Twitter actors that broadcast their messages to a wide audience. Both hubs and gatekeepers were considered 'key actors' within the network. Actors that could act as gatekeepers or hubs were identified by their values of betweenness centrality (i.e., how many actors belonging to different groups a user connects) and in-degree centrality (i.e., how many times a user's posts were retweeted; Himelboim, 2017) since the number of followers is not a good indicator of influence (Cha, Haddadi, Benevenuto, \& Gummadi, 2010; Kwak et al., 2010). Actors meeting these two criteria were more likely to reach diverse audiences and conversations (high betweenness centrality) and to have a high visibility within the network (high in-degree). We did not consider users having high betweenness centrality but in-degree centrality equal to zero and low out-degree centrality (i.e., how many retweets a user made) as key actors, since they were unlikely to have an impact on the conversations.

For each data collection, we identify the top 50 actors having the highest betweenness centrality and/or having an in-degree centrality higher than 20 retweets. Then we excluded from each sample users that had a high centrality value but did not tweet (i.e., they were mentioned in a highly shared tweet), and individuals having high betweenness centrality for interacting with users having a different opinion on vaccinations (e.g., a pro-vaccine user engaged by anti-vaccine actors) or retweeting both anti- and pro-vaccine messages. We identified 48, 46, and 50 key actors in the June, September, and October dataset, respectively.

After identifying these actors, we classified them based on their vaccine sentiment (e.g., pro-vaccine, antivaccine) and type of actor (e.g., activist, parent, health professional). For both classifications we followed Braun and Clarke's (2013) guidelines: We ran a preliminary analysis on the key actors from the first collection (June 2016) to identify potential types of actor and their vaccine sentiment, and we subsequently refined our coding and applied it to all three datasets. To categorise actors into types of actor, we considered the words they had used to describe themselves in their Twitter biography or in the website linked to their profiles. Thus, to classify their vaccine sentiment we evaluated: Twitter biography, names and handles, webpage links, profile and/or background pictures, tweets' content, and hashtags.

We then conducted a small qualitative analysis of the images shared by two anti-vaccine key actors and two pro-vaccine ones to explore the types of images and relative messages that could have the highest popularity within each community. We chose the most retweeted five images for each actor since retweeting can be considered a Twitter practice that shows endorsement of or support for a message (Boyd, Golder, \& Lotan, 2010).

This study received ethical approval by the Faculty Research Ethics Committee of the University of the West of England.

\section{Results and Discussion}

To explore the dissemination of vaccine images on Twitter, we collected tweets having specific parameters three times. We found that, in each collection, most of the tweets were anti-vaccine, whereas only a few tweets reported news, and an even smaller number of tweets were pro-safe vaccine (see Figures 1 and 2). The number of pro-vaccine tweets and academic tweets varied significantly across datasets likely due to the occurrence of specific events, such as conferences or immunisation campaigns. Our results differ from those obtained by previous research. For example, Love et al. (2013) found that the majority of tweets related to vaccinations were neutral, and only a small proportion were anti-vaccine. These differences were likely due to different collection criteria, since we limited the sample of tweets to those having specific hashtags and images, and/or to different coding criteria because we coded the tweets based not only on their textual content but also on their image, hashtag, and embedded links as well.

Twitter users who retweeted anti-vaccine tweets also retweeted pro-safe vaccine messages, whereas actors who shared pro-vaccine posts also shared academic content and news. Hence, we could distinguish two main communities in the overall network: one against vaccination and one in favour. This separation was emphasised by the poor interaction between the two groupsonly one or two actors shared both anti- and pro-vaccine tweets and a few users occasionally engaged with those having a different point of view, though aggressively (see Figure 1). This suggests that vaccine images were mainly shared within insular communities, rather than between them (Himelboim et al., 2017). Moreover, the dissemination of images differed within anti- and pro-vaccine groups. The members of the anti-vaccine group were consistently more connected than the pro-vaccine group: They often retweeted and mentioned each other and did not share outsiders' images. The pro-vaccine network, in- 


\section{COGITATIO}

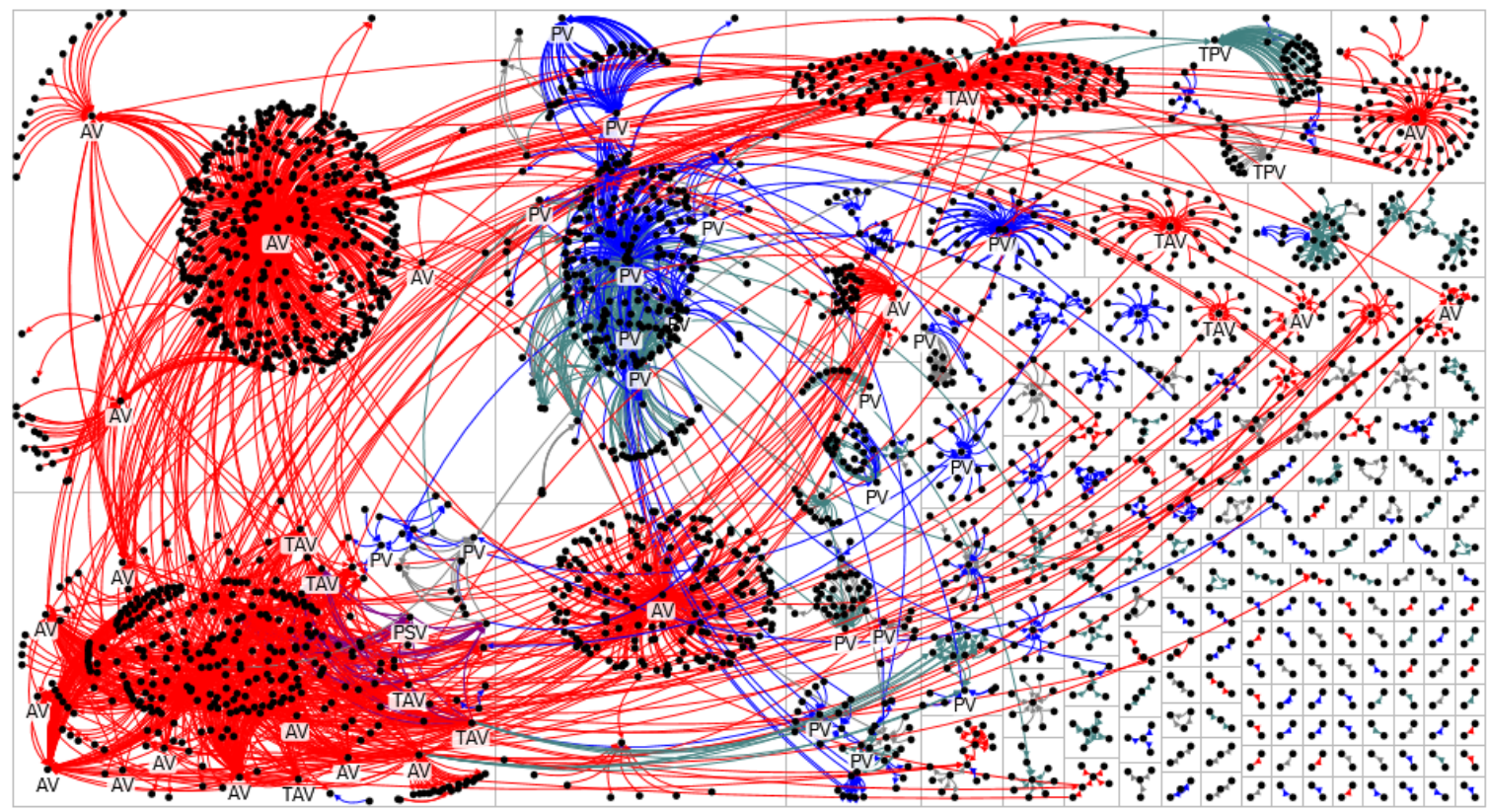

Created with NodeXL Pro (http://nodexl.codeplex.com) from the Social Media Research Foundation (http://www.smrfoundation.org)

Figure 1. Overall network in October 2016. Notes: The arrows indicate the tweets, the dots indicate the users, and the letters indicate the key actors. Red arrows: anti-vaccine tweets; blue arrows: pro-vaccine tweets; grey arrows: news; green arrows: academic tweets; purple arrows: pro-safe vaccine tweets; AV: anti-vaccine actor; PV: pro-vaccine actor; TAV: tendentially anti-vaccine actor; TPV: tendentially pro-vaccine actor.

stead, was always fragmented into several loosely connected clusters, which could favour access to outsiders and new information. Similar results were found by BelloOrgaz et al. (2017) and Yuan and Crooks (2018), who did not find interactions between anti- and pro-vaccine groups on Twitter.

The two communities also had different key actors. In all three datasets, most of these actors belonged to the anti-vaccine group, though in October the number of provaccine actors increased (see Figure 3 ). This could be due to the high number of academic tweets in that dataset, related to the occurrence of a meeting between an NGO and the Islamic Development Bank. This finding contrasts with previous research which observed more pro-vaccine influencers than anti-vaccine on Twitter, though it did not look only at images (Bello-Orgaz et al., 2017). This discrepancy is likely related to both the higher number of anti-vaccine images collected in this study and the criteria used to define key actors. In the next sections, each community and its actors are discussed in detail.

\subsection{The Anti-Vaccine Community}

The anti-vaccine community always had more tweets than the pro-vaccine group, even when it had fewer members; hence, its users likely retweeted antivaccination images more often and were more connected. However, the density and modularity of the community and the dissemination pattern of its images indicate that most of the anti-vaccine users did not retweet

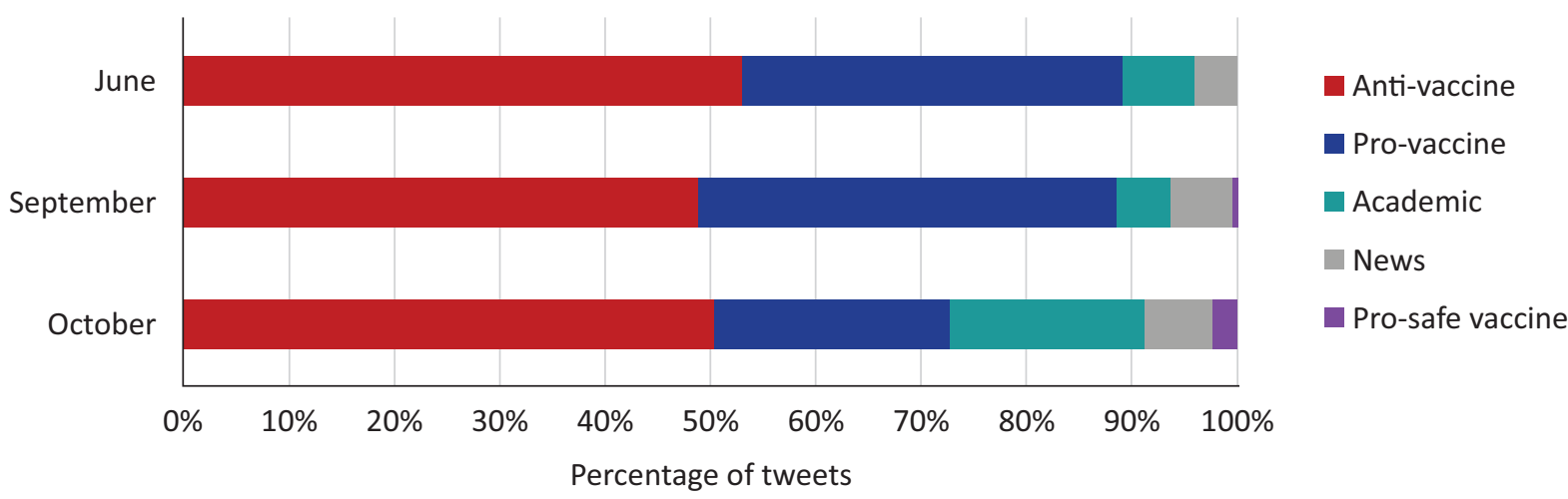

Figure 2. Percentage of tweets for each data collection and each category. Note: The total number of tweets analysed for each collection was 3573 in June, 1932 in September, and 3778 in October. 


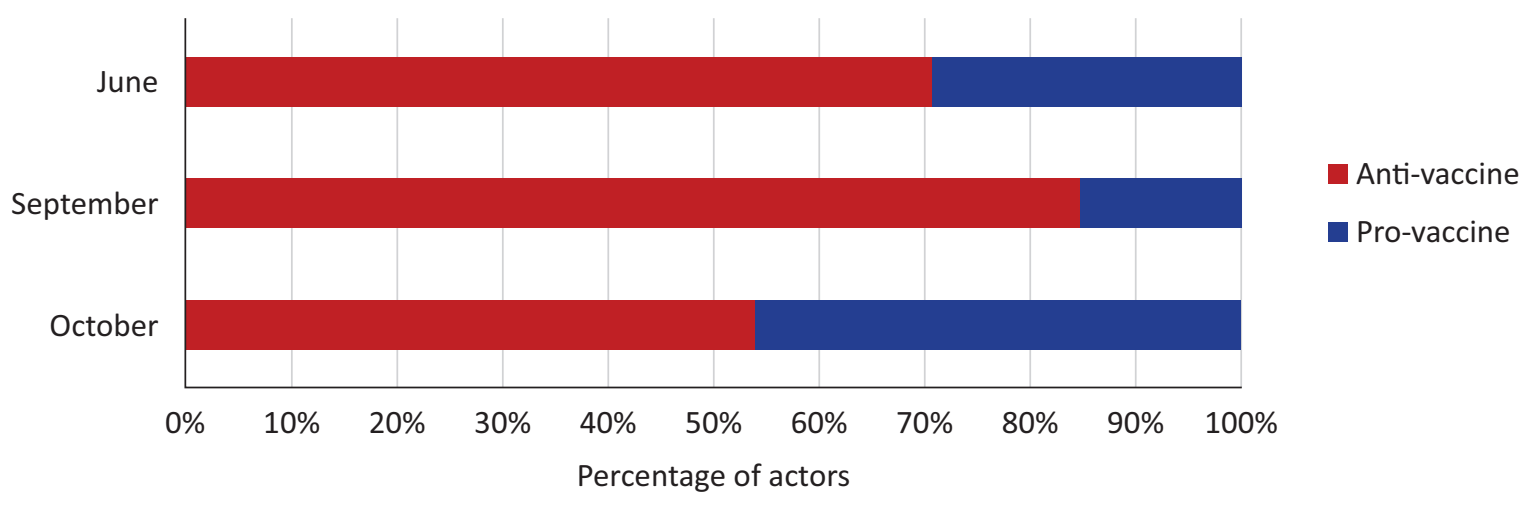

Figure 3. Percentage of anti- and pro-vaccine key actors identified in each dataset. Note: Total number of actors: 48 in June, 46 in September, 50 in October.

each other reciprocally, but clustered around different groups of people (see Table 1 and Figure 1). Therefore, the anti-vaccine visual information was shared mostly within multiple sources and groups (Himelboim et al., 2017). These groups were not disconnected from each other; their members also retweeted other groups' content. The connectivity within and between clusters may indicate that anti-vaccine users valued the information shared by the other members of the network (Himelboim, 2017). Moreover, this connectivity can provide a sense of trust, safety and support to the members of the community (Kadushin, 2011). Previous studies also found that the anti-vaccine community barely engage with outsiders and only re-share its members' tweets (Bello-Orgaz et al., 2017; Himelboim et al., 2019; Yuan \& Crooks, 2018).

Most of the anti-vaccine key actors were activists, parents, parent-activists, and journalist-activists. There were no journalists who were not activists as well. A few of these actors were general users who did not provide any information about themselves (i.e., uncategorised), and even fewer were alternative-health practitioners (see Table 2). As also found by Himelboim et al. (2019), most of the key actors were alternative and nonacademic sources of vaccine visual information. Among them, a journalist-activist and an activist constituted the source of two clusters recurrent in all three datasets (see top-left quadrant and second-to-left bottom quadrant in Figure 1). Several parent-activists, activists, and uncate- gorised users, instead, dominated the flow of visual messages of a third recurrent cluster (see bottom-left quadrant in Figure 1).

These three clusters formed a conspicuous part of the anti-vaccine community. Two of them were broadcasting networks where the actor/hub at their centre (the activist and the activist-journalist) was highly retweeted by the users around them but did not retweet other members of the community (Smith et al., 2014). These two actors disseminated images to their audiences, and they could act as opinion leaders by deciding what visual information to share. Moreover, they may be acknowledged as 'experts' by their audiences who valued and re-shared their content (Murthy, 2012). The other recurrent cluster included most of the other key actors, which frequently retweeted each other thus potentially forming friendship relations and strong ties (Huberman, Romero, \& Wu, 2008). In addition to interacting among themselves, they also retweeted and were retweeted by users belonging to other clusters. This behaviour meant that the shared images reached other groups within the wider anti-vaccine network. Thus, they acted as gatekeepers; they could control the flow of visual information within the anti-vaccine community by choosing the images to share to their audience from other clusters. Moreover, they increased the visibility and redundancy of anti-vaccination images within the whole network, and at the same time, induced social contagion (Harrigan, Achananuparp, \& Lim, 2012). This

Table 1. Number of users and tweets forming the anti- and pro-vaccine networks.

\begin{tabular}{lcccccc}
\hline & \multicolumn{2}{c}{ June } & \multicolumn{2}{c}{ September } & \multicolumn{2}{c}{ October } \\
\cline { 2 - 7 } Graphic metrics & Anti-vaccine & Pro-vaccine & Anti-vaccine & Pro-vaccine & Anti-vaccine & Pro-vaccine \\
\hline Users & 944 & 1056 & 925 & 469 & 1393 & 1135 \\
Tweets & 1896 & 1677 & 1397 & 535 & 2141 & 1637 \\
Density & 0.0021 & 0.0015 & 0.0016 & 0.0024 & 0.0011 & 0.0013 \\
Modularity & 0.49 & 0.72 & 0.71 & 0.92 & 0.66 & 0.80 \\
\hline
\end{tabular}

Notes: The anti-vaccine network includes anti-vaccine and pro-safe vaccine conversations. While tweets were classified into exclusive categories, users were not. The pro-vaccine network includes pro-vaccine, academic tweets, and news. Since a few users were mentioned by, engaged with, or shared content from both anti- and pro-vaccine groups, they were counted in both networks. 
Table 2. Anti- and pro-vaccine key actors classified by type of actor.

\begin{tabular}{|c|c|c|c|c|c|c|}
\hline \multirow[b]{2}{*}{ Type of actors } & \multicolumn{2}{|c|}{ June } & \multicolumn{2}{|c|}{ September } & \multicolumn{2}{|c|}{ October } \\
\hline & $\begin{array}{c}\text { Anti-vaccine } \\
(n=34)\end{array}$ & $\begin{array}{l}\text { Pro-vaccine } \\
(n=14)\end{array}$ & $\begin{array}{l}\text { Anti-vaccine } \\
\quad(n=39)\end{array}$ & $\begin{array}{l}\text { Pro-vaccine } \\
\quad(n=7)\end{array}$ & $\begin{array}{c}\text { Anti-vaccine } \\
(n=27)\end{array}$ & $\begin{array}{c}\text { Pro-vaccine } \\
\quad(n=23)\end{array}$ \\
\hline Activists & $32 \%$ & $0 \%$ & $23 \%$ & $0 \%$ & $33 \%$ & $4 \%$ \\
\hline Parents & $12 \%$ & $0 \%$ & $8 \%$ & $0 \%$ & $7 \%$ & $0 \%$ \\
\hline Parent-Activists & $15 \%$ & $0 \%$ & $10 \%$ & $0 \%$ & $15 \%$ & $0 \%$ \\
\hline Journalist-Activists & $6 \%$ & $0 \%$ & $5 \%$ & $0 \%$ & $4 \%$ & $0 \%$ \\
\hline Alternative Health practitioners & $3 \%$ & $0 \%$ & $5 \%$ & $0 \%$ & $4 \%$ & $0 \%$ \\
\hline Uncategorised & $24 \%$ & $0 \%$ & $26 \%$ & $0 \%$ & $19 \%$ & $0 \%$ \\
\hline NGOs & $0 \%$ & $43 \%$ & $0 \%$ & $14 \%$ & $4 \%$ & $39 \%$ \\
\hline $\begin{array}{l}\text { Chief-Executives, Managers } \\
\text { of NGOs }\end{array}$ & $0 \%$ & $7 \%$ & $0 \%$ & $14 \%$ & $0 \%$ & $13 \%$ \\
\hline Health professionals/Academics & $0 \%$ & $21 \%$ & $3 \%$ & $57 \%$ & $4 \%$ & $35 \%$ \\
\hline Other & $9 \%$ & $29 \%$ & $21 \%$ & $14 \%$ & $11 \%$ & $9 \%$ \\
\hline Total & $100 \%$ & $100 \%$ & $100 \%$ & $100 \%$ & $100 \%$ & $100 \%$ \\
\hline
\end{tabular}

Notes: The category 'Other' includes types of actor that appeared occasionally and not in all three collections (e.g., writer, journalist, public health organization, research centre, pharmaceutical company). Each group (e.g., anti-vaccine) was divided into several categories to show the diversity of type of actors. Hence, the percentages refer to small frequencies.

mechanism might reinforce the echo-chamber effect by excluding information that comes from outside the wellconnected network and reinforcing the messages shared by like-minded members of the community (Southwell, 2013; Yardi \& Boyd, 2010).

We explored the five most retweeted images shared by the two hubs (the activist and the journalist-activist) to gain insights into the messages they conveyed. The activist posted photos saying that vaccines are unsafe. Three of these images included doctors' or medical associations' testimonials supporting these claims, whereas two of them mentioned Vaxxed (documentary) as a reliable source of vaccine information or as a growing anti-vaccination movement in the US. The journalistactivist shared photos or pictures with only textual elements that claimed conspiracy theories behind mandatory vaccinations, suggesting that vaccines are unsafe and cause autism. Both these two hubs shared vaccine misinformation and pseudoscientific evidence. Vaccine safety and conspiracy theories were two common topics of anti-vaccine images shared on Pinterest as well (Guidry et al., 2015).

\subsection{The Pro-Vaccine Network}

The pro-vaccine network had a completely different structure from the anti-vaccine community: It was formed by several loosely connected clusters and it was variable across the three datasets (see Table 1 and Figure 1). However, two clusters that were recurrent across the three datasets linked most of the biggest groups of the pro-vaccine network. These two clusters acted as brokers, reaching out to other groups discussing vaccinations from a slightly different angle (Himelboim et al., 2017). The structure of the pro-vaccine network facilitated access to and diffusion of new or different vi- sual messages, thus avoiding redundancy of information; it also favoured networking, especially among NGOs and foundations who were often key actors (Kadushin, 2011). Previous research found that the pro-vaccine network was better connected, and as in this case, tended to be more open to outsiders than the anti-vaccination community (Bello-Orgaz et al., 2017; Yuan \& Crooks, 2018). The anti- and pro-vaccination communities did not only have a different network structure, but also different types of key actors. The pro-vaccine ones were mainly NGOs, foundations, health professionals, academics, and Chief Executive Officers (CEOs) of NGOs (see Table 2). These actors were more credible sources of information, as also found by Himelboim et al. (2019).

NGOs dominated the flow of visual information in favour of immunisation. Many of them acted as hubs broadcasting their messages to their audiences (Smith et al., 2014). Moreover, an NGO and its CEO were at the centre of two clusters recurrent across the three datasets. These two actors acted as brokers, connecting the other organisations and charities involved in immunisation campaigns; hence, they acted as gatekeepers controlling the dissemination of information among them (Kadushin, 2011). Consistent with a one-way communication flow, it is possible that NGOs saw Twitter primarily as a means to persuade the public of their point of view (Auger, 2013), to create networks of supporters, and for "public education" rather than for mobilisation activities (Guo \& Saxton, 2014). This attitude emerged in the most retweeted images shared by two key pro-vaccine actors: the NGO and its CEO. The NGO shared photos about immunisation campaigns and activities they run, and their partnerships with other non-profit organisations. Their messages did not focus on vaccine safety, but on their efficacy. The images posted by the CEO were photos about the NGO's achievements or charts and in- 
fographics about vaccine efficacy. This actor also shared two infographics related to news about research on vaccine development.

\section{Conclusion}

This study is the first to explore how visual information is disseminated within and among anti- and provaccination networks on Twitter and who the potential gatekeepers are in each community. This research reinforces previous work (Bello-Orgaz et al., 2017) by showing that pro- and anti-vaccine communities do not share images with each other on Twitter. Moreover, the few images explored in this study emphasised the polarisation of these two communities. While the most retweeted anti-vaccine images focused on vaccine safety, vaccine conspiracies, and provided pseudoscientific evidence to support their claims, the pro-vaccine images were about immunisation campaigns, vaccine efficacy, and development. We also found that the pro-vaccine network sharing images split into several loosely connected groups and had NGOs, foundations, healthcare practitioners, and academics as key actors and experts. The structure of this group facilitated networking among non-profit organisations and the exchange of new information about immunisation campaigns and research (Southwell, 2013). The anti-vaccine key actors sharing images were mainly activists or parents or both and were well connected within the network. The high connectivity of this community may reinforce the ties between members and increase their distrust towards non-members. It may also encourage intentions to avoid vaccinating and campaigning against vaccinations (Southwell, 2013). One cluster in particular may have increased the redundancy of visual information within the anti-vaccine network (Harrigan et al., 2012). This redundancy of visual messages, combined with high level of interactions among the members of this cluster, might reinforce the network ties and indirectly encourage those on the margins of the network, who have doubts about vaccinations, to become anti-vaccine as well (Southwell, 2013).

\subsection{Practical Implications}

Anti-vaccine images were predominant. By retweeting each other, anti-vaccination users increased the visibility of their images, enabling them to appear in followers' timelines and the vaccine hashtag streams more often. Hence, these images could potentially reach a broader audience than the pro-vaccine ones (Kumar et al., 2013). Moreover, these images could influence the public not to vaccinate, especially because they were retweeted by activists and parents who may become popular alternative sources of vaccine information (Szomszor, Kostkova, \& Louis, 2011). This could be particularly problematic, as parents using social media to search for vaccine information may place more trust in them than in health professionals (Freed, Clark, Butchart, Singer, \& Davis, 2011).
Pro-vaccine images may not reach as many conversations around hashtags as those against vaccinations do; hence they may not reach as many users who seek vaccine information by searching Twitter hashtags (Bruns \& Burgess, 2015). For visual communication about vaccinations, we suggest targeting those users searching for hashtags such as \#vaccines and \#vaccinations, rather than anti-vaccine actors, as they may be more open to information about immunisation. The World Health Organisation (WHO) has given similar guidance (to target a broad lay public, rather than seek to engage anti-vaccine groups) when speaking at public debates about vaccinations (WHO, 2017). They also suggest correcting vaccine misinformation and unmasking the techniques deniers use to advocate against vaccinations (WHO, 2017).

Trying to persuade anti-vaccine users to vaccinate may not be an effective strategy, as their community is closed and possibly hostile to outsiders (Yuan \& Crooks, 2018). Moreover, they may oppose any content produced by traditional experts and sources of information as they tend to believe in conspiracy theories (Mitra et al., 2016). An alternative approach could be that suggested by Lutkenhaus, Jansz, and Bouman (2019), who mapped vaccine conversations and communities on Twitter and identified their opinion leaders and gatekeepers, in a manner similar to our study. They contacted opinion leaders and gatekeepers at the border of the anti-vaccine communities, who were not deniers nor strong supporters of vaccinations. By engaging with them, and providing correct scientific information and data about vaccines, they were able to reach closed communities who do not trust traditional experts, but will consider provaccine messages discussed by influencers from within the community. Lutkenhaus et al. (2019) also suggested studying the content shared by the target communities. This could include the content and symbols used in images shared by the anti- and pro-vaccine actors (Guidry et al., 2015).

\subsection{Limitations and Future Studies}

The results of this study differ from those of previous research on vaccination networks on Twitter. For example, while we found that most Twitter communities were anti-vaccine, Love et al. (2013) saw that pro-vaccine tweets comprised the majority of the conversation. The high number of anti-vaccine tweets and users we found might be due to our collection criteria. We collected only tweets having pictures, and at least one of ten popular hashtags highly relevant to vaccine discussions, thus excluding tweets having only words such as 'vaccine' that related them to the topic. We took this approach because hashtags, rather than words, are used to actively follow and join a Twitter conversation (Bruns \& Burgess, 2012). Moreover, we considered hashtags such as \#CDCwhistleblower, \#vaxxed, and \#hearus in our collection criteria, which do not contain the word 
'vaccine' but label niche discussions against vaccination. Nevertheless, ten hashtags might not include all the popular conversations on vaccinations. Though the data were collected in 2016, more recent studies have also reported polarisation in vaccine networks (Bello-Orgaz et al., 2017; Yuan \& Crooks, 2018) and observed that the closed nature of the anti-vaccine community could make it difficult to penetrate (Gunaratne et al., 2019). Together with our study, this suggests there may be particular challenges for those undertaking vaccination campaigns on Twitter.

This research contributes to understanding how images about vaccinations flow on Twitter. Further studies are needed to investigate the dynamics within the Twitter anti-vaccine community and the textual and visual content they share since Twitter messages may influence readers not to vaccinate (Dunn et al., 2017). Future research should also focus on vaccine pictures shared on social media in relation to the communities that diffuse them. Moreover, it would be interesting to analyse the differences between network patterns around vaccine hashtags and those around words. Such research would facilitate design of effective Twitter immunisation campaigns, and address the sentiment spread by the antivaccine movement online and offline (Leask, 2015).

\section{Conflict of Interests}

The authors declare no conflict of interests.

\section{References}

All Europe Academies. (2019). Trust in science and changing landscapes of communication (No. 3). Berlin: All European Academies.

Apollonio, D. E., \& Malone, R. E. (2009). Turning negative into positive: Public health mass media campaigns and negative advertising. Health Education Research, 24(3), 483-495.

Auger, G. A. (2013). Fostering democracy through social media: Evaluating diametrically opposed nonprofit advocacy organizations' use of Facebook, Twitter, and YouTube. Public Relations and Democracy, 39(4), 369-376.

Bello-Orgaz, G., Hernandez-Castro, J., \& Camacho, D. (2017). Detecting discussion communities on vaccination in twitter. Future Generation Computer Systems, 66, 125-136.

Betsch, C., Renkewitz, F., Betsch, T., \& Ulshöfer, C. (2010). The influence of vaccine-critical websites on perceiving vaccination risks. Journal of Health Psychology, 15(3), 446-455.

Boyd, D., Golder, S., \& Lotan, G. (2010). Tweet, tweet, retweet: Conversational aspects of retweeting on Twitter. In Proceedings of the 43rd Hawaii International Conference on System Sciences (pp. 1-10). Washington, DC: IEEE Computer Society.

Braun, V., \& Clarke, V. (2013). Successful qualitative re- search: A practical guide for beginners (1st ed.). London: SAGE.

Bruns, A. (2008). Blogs, Wikipedia, second life, and beyond: From production to produsage (2nd ed.). New York, NY: Peter Lang.

Bruns, A., \& Burgess, J. (2012). Researching news discussion on Twitter. Journalism Studies, 13(5/6), 801-814.

Bruns, A., \& Burgess, J. (2015). Twitter hashtags from ad hoc to calculated publics. In N. Rambukkana (Ed.), Hashtag publics: The power and politics of discursive networks (pp. 13-28). New York, NY: Peter Lang.

Bruns, A., \& Moe, H. (2014). Structural layers of communication on Twitter. In K. Weller, A. Bruns, J. Burgess, M. Mahrt, \& C. Puschmann (Eds.), Twitter and society (pp. 15-28). New York, NY: Peter Lang.

Bruns, A., \& Stieglitz, S. (2014). Metrics for understanding communication on Twitter. In K. Weller, A. Bruns, J. Burgess, M. Mahrt, \& C. Puschmann (Eds.), Twitter and society (pp. 69-82). New York, NY: Peter Lang.

Cha, M., Haddadi, H., Benevenuto, F., \& Gummadi, K. P. (2010). Measuring user influence in Twitter: The million follower fallacy. In ICWSM '10: Proceedings of International AAAl Conference on Weblogs and Social (pp. 10-17). Menlo Park, CA: AAAI Press.

Chen, T., \& Dredze, M. (2018). Vaccine images on Twitter: Analysis of what images are shared. Journal of Medical Internet Research, 20(4), e130.

Clauset, A., Newman, M. E. J., \& Moore, C. (2004). Finding community structure in very large networks. Physical Review E, 70(6), 066111.

Del Vicario, M., Bessi, A., Zollo, F., Petroni, F., Scala, A., Caldarelli, G., . . . Quattrociocchi, W. (2016). The spreading of misinformation online. Proceedings of the National Academy of Sciences, 113(3), 554-559.

Dubé, E., Vivion, M., \& MacDonald, N. E. (2015). Vaccine hesitancy, vaccine refusal and the anti-vaccine movement: Influence, impact and implications. Expert Review of Vaccines, 14(1), 99-117.

Duggan, M. (2013). Photo and video sharing grow online. Washington, DC: Pew Research Center.

Dunn, A. G., Leask, J., Zhou, X., Mandl, K. D., \& Coiera, E. (2015). Associations between exposure to and expression of negative opinions about human papillomavirus vaccines on social media: An observational study. Journal of Medical Internet Research, 17(6), e144.

Dunn, A. G., Surian, D., Leask, J., Dey, A., Mandl, K. D., \& Coiera, E. (2017). Mapping information exposure on social media to explain differences in HPV vaccine coverage in the United States. Vaccine, 35(23), 3033-3040.

Freed, G. L., Clark, S. J., Butchart, A. T., Singer, D. C., \& Davis, M. M. (2011). Sources and perceived credibility of vaccine-safety information for parents. Pediatrics, 127(Supp. 1), S107-S112.

Giglietto, F., \& Lee, Y. (2017). A hashtag worth a thousand words: Discursive strategies around \#JeNeSuis- 
PasCharlie after the 2015 Charlie Hebdo shooting. Social Media + Society, 3(1), 1-15.

Grinberg, N., Joseph, K., Friedland, L., Swire-Thompson, B., \& Lazer, D. (2019). Fake news on Twitter during the 2016 U.S. presidential election. Science, 363(6425), 374-378.

Guidry, J. P. D., Carlyle, K., Messner, M., \& Jin, Y. (2015). On pins and needles: How vaccines are portrayed on Pinterest. Vaccine, 33(39), 5051-5056.

Gunaratne, K., Coomes, E. A., \& Haghbayan, H. (2019). Temporal trends in anti-vaccine discourse on Twitter. Vaccine, 37(35), 4867-4871.

Guo, C., \& Saxton, G. D. (2014). Tweeting social change: How social media are changing nonprofit advocacy. Nonprofit and Voluntary Sector Quarterly, 43(1), 57-79.

Harrigan, N., Achananuparp, P., \& Lim, E.-P. (2012). Influentials, novelty, and social contagion: The viral power of average friends, close communities, and old news. Social Networks, 34(4), 470-480.

Himelboim, I. (2017). Social network analysis (Social media). In J. Matthes, C. S. Davis, \& R. F. Potter (Eds.), The international encyclopedia of communication research methods (pp. 1-16). Hoboken, NJ: John Wiley \& Sons.

Himelboim, I., Smith, M. A., Rainie, L., Shneiderman, B., \& Espina, C. (2017). Classifying Twitter topic-networks using social network analysis. Social Media + Society, 3(1), 1-13.

Himelboim, I., Xiao, X., Lee, D. K. L., Wang, M. Y., \& Borah, P. (2019). A social networks approach to understanding vaccine conversations on Twitter: Network clusters, sentiment, and certainty in HPV social networks. Health Communication, 35(5), 607-615.

Houts, P. S., Doak, C. C., Doak, L. G., \& Loscalzo, M. J. (2006). The role of pictures in improving health communication: A review of research on attention, comprehension, recall, and adherence. Patient Education and Counseling, 61(2), 173-190.

Huberman, B. A., Romero, D. M., \& Wu, F. (2008). Social networks that matter: Twitter under the microscope. First Monday, 14(1). https://doi.org/10.5210/ fm.v14i1.2317

Kadushin, C. (2011). Understanding social networks: Theories, concepts, and findings (1st ed.). New York, NY: Oxford University Press.

Kata, A. (2012). Anti-vaccine activists, Web 2.0, and the postmodern paradigm: An overview of tactics and tropes used online by the anti-vaccination movement. Vaccine, 30(25), 3778-3789.

Kumar, S., Morstatter, F., \& Liu, H. (2013). Twitter data analytics. New York, NY: Springer.

Kwak, H., Lee, C., Park, H., \& Moon, S. (2010). What is Twitter, a social network or a news media? In Proceedings of the 19th International Conference on World Wide Web (pp. 591-600). New York, NY: Association for Computing Machinery.

Lama, Y., Chen, T., Dredze, M., Jamison, A., Quinn, S. C., \&
Broniatowski, D. A. (2018). Discordance between human papillomavirus twitter images and disparities in human papillomavirus risk and disease in the United States: Mixed-methods analysis. Journal of Medical Internet Research, 20(9), e10244.

Larson, H. J., Cooper, L. Z., Eskola, J., Katz, S. L., \& Ratzan, S. (2011). Addressing the vaccine confidence gap. The Lancet, 378(9790), 526-535.

Leask, J. (2015). Should we do battle with antivaccination activists? Public Health Research \& Practice, 25(2), e2521515.

LeFebvre, R. K., \& Armstrong, C. (2018). Grievance-based social movement mobilization in the \#Ferguson Twitter storm. New Media \& Society, 20(1), 8-28.

Love, B., Himelboim, I., Holton, A., \& Stewart, K. (2013). Twitter as a source of vaccination information: Content drivers and what they are saying. American Journal of Infection Control, 41(6), 568-570.

Lutkenhaus, R. O., Jansz, J., \& Bouman, M. P. (2019). Tailoring in the digital era: Stimulating dialogues on health topics in collaboration with social media influencers. Digital Health, 5, 2055207618821521.

Meadows, C. Z., Tang, L., \& Liu, W. (2019). Twitter message types, health beliefs, and vaccine attitudes during the 2015 measles outbreak in California. American Journal of Infection Control, 47(11), 1314-1318.

Mitra, T., Counts, S., \& Pennebaker, J. W. (2016). Understanding anti-vaccination attitudes in social media. In Proceedings of the 10th International Conference on Web and Social Media (pp. 269-278). Menlo Park, CA: AAAI Press.

Murthy, D. (2012). Twitter: Social communication in the Twitter age (1st ed.). Cambridge: Polity Press.

Newman, M. (2010). Networks: An introduction. New York, NY: Oxford University Press.

Scanfeld, D., Scanfeld, V., \& Larson, E. L. (2010). Dissemination of health information through social networks: Twitter and antibiotics. American Journal of Infection Control, 38(3), 182-188.

Schmidt, J.-H. (2014). Twitter and the rise of personal publics. In K. Weller, A. Bruns, J. Burgess, M. Mahrt, \& C. Puschmann (Eds.), Twitter and society (pp. 3-14). New York, NY: Peter Lang.

Smith, M. A., Rainie, L., Shneiderman, B., \& Himelboim, I. (2014). Mapping Twitter topic networks: From polarized crowds to community clusters. Washington, DC: Pew Research Center.

Southwell, B. G. (2013). Social networks and popular understanding of science and health: Sharing disparities (1st ed.). Baltimore, MD: Johns Hopkins University Press.

Szomszor, M., Kostkova, P., \& Louis, C. S. (2011). Twitter informatics: Tracking and understanding public reaction during the 2009 swine flu pandemic. In Proceedings of the 2011 IEEE/WIC/ACM International Conferences on Web Intelligence and Intelligent Agent Technology (pp. 320-323). Washington, DC: IEEE Computer Society. 
Vosoughi, S., Roy, D., \& Aral, S. (2018). The spread of true and false news online. Science, 359(6380), 1146-1151.

World Health Organisation. (2017). Best practice guidance: How to respond to vocal vaccine deniers in public. Copenhagen: World Health Organisation.

Wilson, K., Atkinson, K., \& Deeks, S. (2014). Opportunities for utilizing new technologies to increase vaccine confidence. Expert Review of Vaccines, 13(8), 969-977.

Witteman, H. O., \& Zikmund-Fisher, B. J. (2012). The defining characteristics of Web 2.0 and their potential influence in the online vaccination debate. Vaccine, 30(25), 3734-3740.
Xu, W. W., Chiu, I.-H., Chen, Y., \& Mukherjee, T. (2015). Twitter hashtags for health: Applying network and content analyses to understand the health knowledge sharing in a Twitter-based community of practice. Quality \& Quantity, 49(4), 1361-1380.

Yardi, S., \& Boyd, D. (2010). Dynamic debates: An analysis of group polarization over time on Twitter. Bulletin of Science, Technology \& Society, 30(5), 316-327.

Yuan, X., \& Crooks, A. T. (2018). Examining online vaccination discussion and communities in Twitter. In Proceedings of the 9th International Conference on Social Media and Society (pp. 197-206). New York, NY: Association for Computing Machinery.

\section{About the Authors}

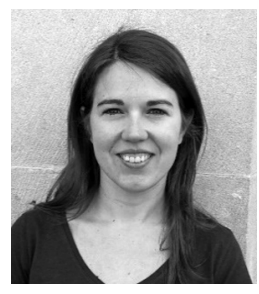

Elena Milani is a Research Fellow for the European Project RETHINK and a PhD candidate in Science Communication at the University of the West of England (UWE Bristol). Her research interests focus on digital and visual communication of science and health. Elena is especially interested in the produsage and dissemination of digital images about vaccines in social media communities. She also teaches about these topics in postgraduate degrees in Science Communication at UWE Bristol and the University of Trento (Italy).

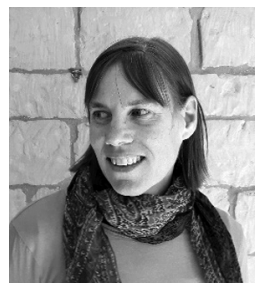

Emma Weitkamp (PhD) is an Associate Professor in Science Communication at UWE Bristol, where she is Programme Leader for the Postgraduate Diploma in Applied Science Communication (online). Emma's research interests focus on the actors involved in communicating science, particularly those that play intermediary roles between scientists and broader publics. She is particularly interested in the roles these actors play and their perspectives and identities as science communicators.

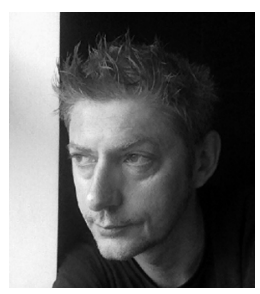

Peter Webb (PhD) is a Writer, Lecturer, and Musician who specialises in research into popular and contemporary music, subcultures, politics, and social theory. He is a Senior Lecturer in Sociology at the UWE Bristol. Webb previously worked within an independent record label from 1990-2002 as an Artist and Tour Manager, the physical theatre companies Blast Theory and Intimate Strangers and the film company Parallax Pictures. He is the Owner and Creative Director of the publishing company PC-Press. 